Phys. Rev. B 68, 144519 (2003)

\title{
Electronic Collective Modes and Superconductivity in Layered Conductors
}

\author{
A. Bill \\ Paul Scherrer Institute, Condensed Matter Theory, 5232 Villigen PSI, Switzerland \\ H. Morawitz \\ IBM Almaden Research Center, 650 Harry Rd., San Jose, CA 95120, USA, and \\ Institute for Theoretical Physics, Ulm University, 89069 Ulm, Germany \\ V.Z. Kresin \\ Lawrence Berkeley Laboratory, University of California, Berkeley, CA 94720, USA
}

(Dated: October 24, 2018)

\begin{abstract}
A distinctive feature of layered conductors is the presence of low-energy electronic collective modes of the conduction electrons. This affects the dynamic screening properties of the Coulomb interaction in a layered material. We study the consequences of the existence of these collective modes for superconductivity. General equations for the superconducting order parameter are derived within the strong-coupling phonon-plasmon scheme that account for the screened Coulomb interaction. Specifically, we calculate the superconducting critical temperature $T_{c}$ taking into account the full temperature, frequency and wave-vector dependence of the dielectric function. We show that low-energy plasmons may contribute constructively to superconductivity. Three classes of layered superconductors are discussed within our model: metal-intercalated halide nitrides, layered organic materials and high- $T_{c}$ oxides. In particular, we demonstrate that the plasmon contribution (electronic mechanism) is dominant in the first class of layered materials. The theory shows that the description of so-called "quasi-two-dimensional superconductors" cannot be reduced to a purely $2 \mathrm{D}$ model, as commonly assumed. While the transport properties are strongly anisotropic, it remains essential to take into account the screened interlayer Coulomb interaction to describe the superconducting state of layered materials.
\end{abstract}

PACS numbers:

\section{INTRODUCTION}

Recent years have witnessed the discovery of many new superconducting materials: high-temperature cuprates, fullerides, borocarbides, ruthenates, $\mathrm{MgB}_{2}$, metalintercalated halide nitrides, intercalated $\mathrm{Na}_{x} \mathrm{CoO}_{2}{ }^{1}$, etc. Systems such as organics, heavy fermions, nanoparticles have also been intensively studied. Many of these systems belong to the family of layered conductors characterized, e.g., by strongly anisotropic electronic transport properties. Recently, it was reported that even $\mathrm{Ba}_{1-x} \mathrm{~K}_{x} \mathrm{BiO}_{3}$ has a layered structure $2^{2}$ An interesting question raised by the observation of superconductivity in all the systems mentioned above is the following: why is layering a favorable factor for superconductivity? The present paper addresses this question. We show that layering leads to peculiar dynamic screening of the Coulomb interaction and that this is important for the description of the superconducting state in layered conductors.

The conventional theory of superconductivity has mostly dealt with three-dimensional (3D) isotropic systems, although some papers have also described the impact of the Fermi surface anisotropy on the superconducting state (see, e.g., review Ref. 3 ). In this theory the Coulomb repulsion is described by a static pseudopotential $\mu^{\star}$ and its value is reduced because of the well-known logarithmic factor $\ln (E / \Omega)$ where $E$ is an electronic energy and $\Omega$ is a characteristic bosonic (e.g. phonon) energy. Such a static approach is justified by the large value of the plasmon frequency $\Omega_{p l}(\mathbf{q}=0)=\min \{\Omega(\mathbf{q})\} \equiv \Omega_{p l}$ in usual metals, where $\Omega_{p l}$ ranges between $5 \mathrm{eV}$ and $30 \mathrm{eV}$. Such high energies imply a perfect, instantaneous screening of the Coulomb interaction.

Layered conductors have a structure of the plasmon spectrum that differs fundamentally from 3D metals. In addition to the high energy "optical" collective mode mentioned above, the spectrum contains also an important low-frequency part (see below). The screening of the Coulomb interaction is incomplete and the dynamic nature of the interaction becomes important. As a result, the interplay between the attractive interaction and the Coulomb term is more subtle than introduced in the conventional theory of superconductivity. It is on this screened Coulomb term and its interplay with the electron-phonon mechanism that we focus in the present paper.

Our goal is to evaluate the additional impact of dynamic screening on pairing in layered superconductors. The pure plasmon mechanism (that is, in absence of any other attractive interaction) has been discussed previously for 3D and 2D systems (see, e.g., Refs. 4 5 6. 7) The acoustic plasmons for spatially separated layers in metaloxide semiconductor structures were introduced and analyzed by Y. Takada in Ref. 8. The author indicated the possibility of acoustic-plasmon mediated superconductivity. In the present paper we focus on layered conductors. More importantly, we consider plasmons' contribution in conjunction with the phonon mechanism. It is assumed 
that the phonons themselves provide the pairing so that at $T=0 \mathrm{~K}$ the compound is in the superconducting state. In other words, the presence of phonons is sufficient to overcome the static Coulomb repulsive interaction. Within this scenario the dynamic screening acts as an additional factor. Therefore, in the absence of the plasmon term we obtain the conventional Eliashberg equations; the electron-phonon coupling constant and the Coulomb pseudopotential are thus considered as parameters to be determined from experimental data (see, e.g., Ref. 9). Note that the contribution of phonons and plasmons to the superconducting state has aslo been considered in Ref. 10 for fullerides. We also point out that we consider the electron-phonon interaction (phonon-plasmon mechanism) for concreteness. However, since our attention is set on the Coulomb contribution to the total pairing, our approach is valid for other mechanisms as well. The advantage of the present approach is that we are not restricted to answer the question whether or not plasmons themselves can provide superconductivity, but allows to answer the question whether low-energy plasmons can sustain or enhance the pairing induced by other mechanisms.

We discussed briefly our approach in Refs. 11, $12,1314$. The present article contains a detailed analysis of the dielectric function, the plasmon spectrum and its impact on superconductivity in layered superconductors. Furthermore, we apply the theory to characteristic examples of three classes of materials: the metal-intercalated halide nitrides, organic and high-temperature superconductors.

The structure of the paper is as follows. In section 1 we present the main equations describing layered superconductors and discuss the electron-phonon and Coulomb contributions to the pairing-interaction kernel. In section III we discuss the dynamic screening of the Coulomb interaction in layered conductors. The dielectric function and the resulting electronic collective excitations (layer plasmons) will be described. It is essential that the dielectric function is evaluated and analyzed in the thermodynamic Green's function formalism; this allows us to calculate $T_{c}$. In the next section, Sec. IV we consider three classes of layered superconductors: the metalintercalated halide nitrides, the organics and high- $T_{c}$ superconductors. The conclusions are presented in Sec. $\nabla$

\section{MAIN EQUATIONS}

We consider a layered system consisting of stacks of conducting sheets along the $z$-axis separated by dielec- tric spacers (see Fig. 11). Because of the conductivity's high anisotropy it is a good approximation to neglect transport between the layers (see Sec. IV]. On the other hand, the Coulomb interaction between charge-carriers is effective both within and between the sheets. To ensure charge-neutrality we further introduce positive countercharges spread out homogeneously over the sheets.

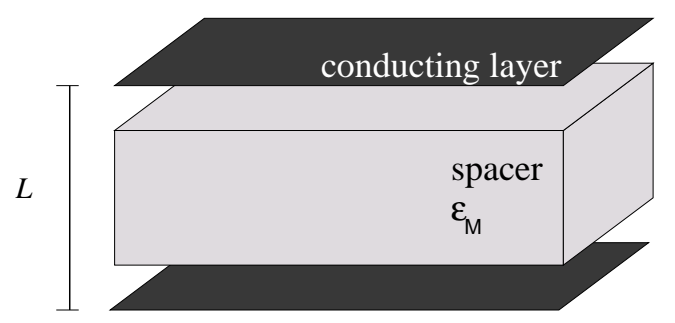

FIG. 1: The layered electron gas (LEG) model. The conducting sheets (dark) are stacked along $c$ and separated by spacers (light) with dielectric constant $\epsilon_{M}$. The model considers an infinite stacking of layers. The electrons are moving within the conducting sheets. The Coulomb interaction is effective within, but also between the sheets (see text). $L$ is the interlayer distance.

In order to calculate the critical temperature $T_{c}$ for the superconducting transition we start with the equation for the superconducting order parameter $\Delta\left(\mathbf{k}, \omega_{n}\right)$ and the renormalization function $Z\left(\mathbf{k}, \omega_{n}\right)$ :

$$
\begin{gathered}
\Delta\left(\mathbf{k}, \omega_{n}\right) Z\left(\mathbf{k}, \omega_{n}\right)= \\
T \sum_{m=-\infty}^{\infty} \int \frac{\mathrm{d}^{3} \mathbf{k}^{\prime}}{(2 \pi)^{3}} \Gamma\left(\mathbf{k}, \mathbf{k}^{\prime} ; \omega_{n}-\omega_{m}\right) F^{\dagger}\left(\mathbf{k}^{\prime}, \omega_{m}\right) \\
Z\left(\mathbf{k}, \omega_{n}\right)-1= \\
\frac{T}{\omega_{n}} \sum_{m=-\infty}^{\infty} \int \frac{\mathrm{d}^{3} \mathbf{k}^{\prime}}{(2 \pi)^{3}} \Gamma\left(\mathbf{k}, \mathbf{k}^{\prime} ; \omega_{n}-\omega_{m}\right) G\left(\mathbf{k}^{\prime}, \omega_{m}\right)
\end{gathered}
$$

where $F^{\dagger}=<c_{\mathbf{k}, \uparrow}^{\dagger} c_{-\mathbf{k}, \downarrow}^{\dagger}>$ is the Gor'kov pairing function, $G=<c_{\mathbf{k}, \sigma}^{\dagger} c_{\mathbf{k}, \sigma}>$ is the usual Green function, and $\Gamma$ is the total interaction kernel.

These equations can be rewritten in the following form (at $T=T_{c}$ ) 


$$
\begin{aligned}
\phi_{n}(\mathbf{k}) & =\left.T \sum_{m=-\infty}^{\infty} \int \frac{\mathrm{d}^{3} \mathbf{k}^{\prime}}{(2 \pi)^{3}} \Gamma\left(\mathbf{k}, \mathbf{k}^{\prime} ; \omega_{n}-\omega_{m}\right) \frac{\phi_{m}\left(\mathbf{k}^{\prime}\right)}{\omega_{m}^{2}\left(\mathbf{k}^{\prime}\right)+\xi_{\mathbf{k}^{\prime}}^{2}}\right|_{T_{c}}, \\
\omega_{n}(\mathbf{k})-\omega_{n} & =\left.T \sum_{m=-\infty}^{\infty} \int \frac{\mathrm{d}^{3} \mathbf{k}^{\prime}}{(2 \pi)^{3}} \Gamma\left(\mathbf{k}, \mathbf{k}^{\prime} ; \omega_{n}-\omega_{m}\right) \frac{\omega_{m}\left(\mathbf{k}^{\prime}\right)}{\omega_{m}^{2}\left(\mathbf{k}^{\prime}\right)+\xi_{\mathbf{k}^{\prime}}^{2}}\right|_{T_{c}} .
\end{aligned}
$$

In these equations and in the rest of the paper we use the following notations: $\mathbf{k}=\left(\mathbf{k}_{\|}, k_{z}\right)$, where the $z$-axis is chosen to be perpendicular to the layers. We use the thermodynamic Green's function formalism (see, e.g., Ref. 15) with $\omega_{n}=(2 n+1) \pi T$. Because of the relation $\omega_{n}-\omega_{m}=2 \pi T(n-m)$ we often use the short hand $(n-m)$ to denote the frequency dependence [e.g., $(n+m+1)$ stands for $\left.\omega_{n}-\omega_{-(m+1)}\right]$. Finally, we define $\phi_{n}(\mathbf{k}) \equiv \Delta_{n}(\mathbf{k}) Z_{n}(\mathbf{k}), \omega_{n}(\mathbf{k}) \equiv \omega_{n} Z_{n}(\mathbf{k}), \Delta_{n} \equiv \Delta\left(\omega_{n}\right)$ and $Z_{n} \equiv Z\left(\omega_{n}\right)$.

For concreteness we focus on the case where the interaction kernel is a sum of electron-phonon and Coulomb interactions. Then, the total kernel $\Gamma \equiv \Gamma\left(\mathbf{q}, \omega_{n}-\omega_{m}\right)$, with $\mathbf{q}=\mathbf{k}-\mathbf{k}^{\prime}$, is written in the form

$$
\Gamma=\Gamma_{p h}+\Gamma_{c}
$$

with

$$
\begin{aligned}
\Gamma_{p h}(\mathbf{q} ;|n-m|) & =\left|g_{\nu}(\mathbf{q})\right|^{2} D(\mathbf{q},|n-m|) \\
& =\left|g_{\nu}(\mathbf{q})\right|^{2} \frac{\Omega_{\nu}^{2}(\mathbf{q})}{\left(\omega_{n}-\omega_{m}\right)^{2}+\Omega_{\nu}^{2}(\mathbf{q})} \\
\Gamma_{c}(\mathbf{q} ;|n-m|) & =\frac{V_{c}(\mathbf{q})}{\epsilon(\mathbf{q},|n-m|)} .
\end{aligned}
$$

$D(\mathbf{q}, n-m)$ is the phonon Green function, $\Omega_{\nu}^{2}(\mathbf{q})$ the phonon dispersion relation; summation over phonon branches $\nu$ is assumed. The second, important Coulomb term $\Gamma_{c}$ is written in its most general form as the ratio of the bare Coulomb interaction $V_{c}(\mathbf{q})$ and the dielectric function $\epsilon\left(\mathbf{q}, \omega_{n}-\omega_{m}\right)$. Both the Coulomb interaction and the dielectric function have to be calculated for a layered structure. It should be noted that the relation between these two interactions $\left[\Gamma_{p h}\right.$ and $\Gamma_{c}$, Eq. (3) $]$ is more subtle. For example, the Coulomb screening affects the value of the electron-phonon matrix elements (see, e.g., Ref. 16). Here, however, we do not calculate the electron-phonon coupling constant $\lambda$ [see Eq. (19a)] and, similarly to the treatment of conventional superconductors (see, e.g., Ref. 9), we use the values determined from experimental data. For example, in the case halide nitrides considered in Sec. IVB $\lambda$ was determined from heat capacity measurements 18 .

The Coulomb potential $V_{c}(\mathbf{q})$ is the Fourier transform of the 3D Coulomb interaction $V_{c}(\mathbf{r})=e^{2} / \epsilon_{M}|\mathbf{r}|$, where $\epsilon_{M}$ is the dielectric constant of the spacers, and takes the form (see appendix A

$$
V_{c}(\mathbf{q})=\frac{2 \pi e^{2}}{\epsilon_{M} q_{\|}} R\left(q_{\|}, q_{z}\right)
$$

where $R\left(q_{\|}, q_{z}\right)$ is defined in Eq. (8) below. Introducing dimensionless quantities $\tilde{q}=q_{\|} / 2 k_{F}, \kappa_{F}=2 k_{F} L(L$ is the interlayer distance, $k_{F}$ the in-plane Fermi wavevector) as well as $N(0)=m_{b} / 2 \pi \hbar^{2}$, the $2 \mathrm{D}$ electronic density of states $\left(m_{b}\right.$ is the band mass), we can write

$$
V_{c}(\mathbf{q})=\frac{\lambda_{c}}{N(0)} \frac{R\left(\tilde{q} ; q_{z}\right)}{\tilde{q}}
$$

with

$$
R\left(\tilde{q}, q_{z}\right)=\frac{\sinh \left(\kappa_{F} \tilde{q}\right)}{\cosh \left(\kappa_{F} \tilde{q}\right)-\cos \left(q_{z} L\right)}\left(1-\delta_{\mathbf{q}, 0}\right) .
$$

Eq. (6) contains the product of the two-dimensional Coulomb potential and the function $R\left(q_{\|}, q_{z}\right)$ which reflects the layered nature of the studied system. As expected, $\lim _{L \rightarrow \infty} R(\mathbf{q})=1$, whereas $R(\mathbf{q})=2 q_{\|} /|\mathbf{q}|^{2} L$ for $L \ll 1$. Furthermore, $V_{c}$ diverges as $1 /|\mathbf{q}|^{2}$ for $|\mathbf{q}| \rightarrow 0$, in agreement with the $3 \mathrm{D}$ character of this limit. Note that $R\left(\tilde{q} ; q_{z}\right)$ contains the factor $\left(1-\delta_{\mathbf{q}, 0}\right)$ reflecting the presence of the neutralizing positive ion counter-charges; the presence of this term is implicit in the following.

Eq. (7) contains the dimensionless Coulomb interaction constant defined by

$$
\lambda_{c}=\frac{1}{2 \epsilon_{M}}\left(\frac{e^{2}}{\hbar v_{F}}\right)=\frac{r_{s}}{\sqrt{8}}=\frac{\alpha}{2} \frac{c}{v_{F}} .
$$

$v_{F}$ is the Fermi velocity, $c$ the vacuum speed of light and $\alpha$ is the fine structure constant. Note that $\lambda_{c} \sim r_{s}$, where $r_{s}=\sqrt{2} / k_{F} r_{B}\left(r_{B}=\hbar^{2} \epsilon_{M} / m e^{2}\right.$ is the Bohr radius $)$ is the well-known dimensionless electron density radius defined here for a layered electron gas.

The electronic screening of the Coulomb interaction is described by the dielectric function $\epsilon\left(\mathbf{q}, \omega_{n}\right)$ written in its most general form as

$$
\epsilon\left(\mathbf{q}, \omega_{n}-\omega_{m}\right)=1-V_{c}(\mathbf{q}) \Pi\left(\mathbf{q}, \omega_{n}-\omega_{m}\right) .
$$

In the following we use the RPA method. As is known (see, e.g., Ref. 17) for real 3D metals the RPA provides a qualitative description, whereas a quantitative analysis requires to go beyond this approximation. For the systems of interest RPA is favorable because of the inequality $\lambda_{c}<1$ (see below). Note that the contribution of the background dielectric function and the inequality $\epsilon_{M}>1$ could be essential (cf. e.g. Ref. 19). It would be interesting to perform more exact calculations using 
methods as those of Ref. 20 and, in addition, take into account the band structure of real materials instead of the LEG model. We think that the approximation based on the inequality $\lambda_{c}<1$ provides the adequate physical picture.

\section{LAYERED CONDUCTORS: ELECTRONIC COLLECTIVE MODES}

\section{A. Plasmon bands}

The spectrum of collective electronic excitations is determined by the poles of the two-particle Green function which coincides with the poles of the vertex $\Gamma_{c}(\mathbf{q}, \omega)$. The latter is the analytic continuation (see, e.g., Ref. 15) of the function $\Gamma_{c}\left(\mathbf{q}, \omega_{n}\right)$, Eq. (5). These poles correspond to the zeros of the real-frequency dielectric function $1-V_{c}(\mathbf{q}) \Pi\left(\mathbf{q}_{\|}, \omega\right)=0$. At $T=0$ the real part of the polarizability of a single layer takes the form $\left(\omega>\hbar q_{\|} v_{F}\right)^{12.13}$

$$
\operatorname{Re}\{\Pi(\mathbf{q}, \omega)\}=2 N(0)\left[\frac{\omega}{\sqrt{\omega^{2}-\left(\hbar q_{\|} v_{F}\right)^{2}}}-1\right] .
$$

For $\omega \gg \hbar q_{\|} v_{F}$ this expression reduces to $\operatorname{Re}\{\Pi\} \simeq$ $N(0) \hbar^{2} q_{\|}^{2} v_{F}^{2} / \omega^{2}$, as obtained in Ref. 13 .

From Eqs. (17), (8) and (11) we derive the general expression for the plasmon dispersion relation:

$$
\omega=\hbar q_{\|} v_{F} \sqrt{1+\frac{\left(N(0) V_{c}\right)^{2}}{\frac{1}{4}+N(0) V_{c}}},
$$

where $V_{c} \equiv V_{c}\left(\tilde{q}, q_{z}\right)$ is the Coulomb interaction defined in Eq. (7). If $N(0) V_{c} \gg 1$ we obtain the optical plasmon $\omega=\hbar q_{\|} v_{F} \sqrt{1+N(0) V_{c}}$ (this corresponds to the hydrodynamic approximation for small q; see Ref. 21). The plasmon band $\omega=\omega\left(q_{\|}, q_{z}\right)$ is confined between the upper branch with $q_{z}=0$ (in-phase motion of the charge carriers) and the lower branch at $q_{z}=\pi / L$ (outof-phase motion of carriers). Indeed, for $\omega \gg \hbar q_{\|} v_{F}$ Eq. (12) reduces to the expression $\omega \simeq \hbar q_{\|} v_{F} \sqrt{N(0) V_{c}}$ which at $q_{z}=0$ leads to the usual "optical" plasmon with $\Omega_{p l}^{2} \equiv \omega^{2}\left(q_{\|}=0, q_{z}=0\right)=4 e^{2} \varepsilon_{F} / \epsilon_{M} L$. For $q_{z}=\pi / L$, on the other hand, we obtain the dispersion law for the "acoustic" plasmon (linear in $q_{\|}$) of the form of Eq.(12) with

$$
N(0) V_{c}\left(\tilde{q}, q_{z}=\frac{\pi}{L}\right)=\frac{\lambda_{c}}{\tilde{q}} \frac{\sinh \left(\kappa_{F} \tilde{q}\right)}{\cosh \left(\kappa_{F} \tilde{q}\right)+1} \stackrel{q_{\|} \rightarrow 0}{\longrightarrow} \lambda_{c} k_{F} L(.13)
$$

For $q_{z}=\pi / L$ and $q_{\|} L \ll 1$, we obtain $\omega \approx\left(\Omega_{p l} L / 2\right) q_{\|}$.

Thus, the plasmon spectrum of a layered conductor, Eq. (12), has the rather complicated structure shown in Fig. [2]

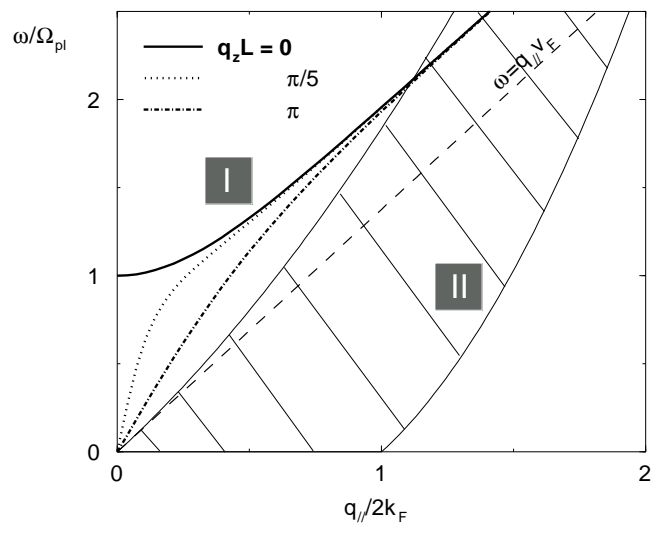

FIG. 2: Electronic excitation spectrum for the layered electron gas (LEG) (see also Ref. 13). The solid, dotted and dash-dotted lines in region I $\left(\omega>\hbar q_{\|} v_{F}\right)$ are plasmon dispersion relations corresponding to the $q_{z}$ indicated on the figure. The area delimited by the $q_{z}=0$ (solid) and $q_{z}=\pi / L$ (dash-dotted) bands contains the dispersion relations for all $q_{z}$. The branch $q_{z}=\pi / 5 L$ (dotted) is shown as an example. The hashed area denotes the electron-hole excitation continuum in which plasmon (Landau) damping occurs. The long-dashed line separates region I $\left(\omega>\hbar q_{\|} v_{F}\right)$ from region II $\left(\omega<\hbar q_{\|} v_{F}\right)$.

The dispersion can be viewed as a continuous set of "acoustic" branches parametrized by $q_{z} \in[0, \pi / L]$ (the slope of the acoustic plasmon at $q_{\|} \rightarrow 0$ is smallest for $q_{z}=\pi / L$ and increases as $q_{z} \rightarrow 0$ ). Only the upper branch for $q_{z}=0$ represents an "optical" branch and, as expected for the long wavelength limit, corresponds to the usual 3D plasmon. Crucial for the phenomenon of dynamic screening and its impact on the pairing is the presence of the low-energy collective excitations, which can play a role similar to phonons (they can be labeled "electronic" sound).

Note that the low-energy plasmon branches, so-called "demons", also appear in the presence of different overlapping bands (e.g. "light" and "heavy" carriers; see, Ref. 4 and the review Ref. 66). We emphasize that the case considered in the present paper is entirely different. Indeed, the appearance of "acoustic" branches is caused by the presence of spatially separated conducting layers and the out-of-phase motion of the carriers in neighbouring planes.

The partial density of states can be determined for each plasmon band (corresponding to each $q_{z}$ ) from the dispersion relation, Eq. (12). As first pointed out by two of the authors, Bozovic et al. in Ref. 13, the density of states considered as a function of energy is peaked at the boundaries, that is for $q_{z}=0$ and $q_{z}=\pi / L$. A good approximation is thus to model the plasmon spectrum of a layered conductor as consisting of two branches: the upper "optical" branch $\left(q_{z}=0\right)$ and the lower "acoustic" branch $\left(q_{z}=\pi / L\right)$. We have shown earlier 14 that the optical branch gives an essentially repulsive contribution to the pairing interaction. We therefore include 
this latter part in the effective repulsive $\mu^{\star}$ of region II (Fig. 2). In the following we consider only the contribution of the dominant acoustic branch at $q_{z}=\pi / L$. The contribution of all other branches only enhances the effect of the $q_{z}=\pi / L$ branch, as we discuss below. It is worth emphasizing that the existence of the latter branches is specific to layered materials.

We end this section by noting that the inclusion of a residual interlayer hopping would imply the appearence of a small gap for the acoustic plasmons. The size of this gap is determined by the interplane hopping parameter $t_{z}$. The more isotropic the system becomes, the larger is the gap. In the isotropic limit the one degenerate optical plasmon branch observed in 3D metals is recovered. As mentioned in the introduction, however, the materials of interest for the present paper (Sec. IV) have a ratio $t_{z} / t_{\|} \lesssim 10^{-3}$ so that discarding interlayer transport is a good approximation. Further support for this approximation is found in Ref. 22 23 (and references therein) from dielectric properties and lattice dynamics studies of high-temperature superconductors.

\section{B. Screening of the Coulomb interaction: the dielectric function at finite temperature.}

To study the impact of dynamic screening on the superconducting state we need to calculate the dielectric function, Eq. (10), which contains the polarizability $\Pi\left(\mathbf{q}, \omega_{n}\right)$. In particular, to obtain $T_{c}$, we have to determine these functions at finite temperatures. In RPA the polarizability takes the well-known form

$$
\Pi\left(\mathbf{q}_{\|}, i \omega_{n}\right)=2 \int d^{2} \mathbf{k}_{\|} \frac{f_{\mathbf{k}_{\|}}-f_{\mathbf{k}_{\|}+\mathbf{q}_{\|}}}{i \omega_{n}+\xi_{\mathbf{k}_{\|}}-\xi_{\mathbf{k}_{\|}+\mathbf{q}_{\|}}} .
$$

where $f_{k} \equiv f\left(\xi_{\mathbf{k}_{\|}}\right)$is the Fermi distribution and all wave vectors lie in the plane of the layered structure. To the best of our knowledge all previous works concerned with layered structures were done either using the calculated polarizability at $T=0$ (see, e.g., Ref. 24) or taking the static limit for non-zero temperatures (as done, e.g., in Ref. 4,5) Here we calculate the polarizability both at finite temperatures (using the temperature Green's function formalism) and all values of $\left(\mathbf{q}, \omega_{n}\right)$. As the calculations of the next sections will show, the temperature dependence of the polarizability can be neglected in some cases (e.g. for halide nitrides, Sec. IVB) but should be taken into account for the cuprates where the ration $T_{c} / \varepsilon_{F}$ is not negligibly small (see Sec. IVD). In general, the proper account of dynamic screening requires to consider all three parameters. Note that to render the numerical problem tractable when solving Eq. (17) below, we reduce the number of integrals to be performed numerically by writing Eq. (14) in polar coordinates and integrating analytically over the angles (cf. appendix $\mathbb{B}$ ). The remaining $k$-integration is then done numerically.

Fig. 3 displays the polarizability, Eq. (14), of the electron gas of a layer, as a function of wave-vector $\tilde{q}=q_{\|} / 2 k_{F}$ for different values of the frequency $\omega_{n}$ and a typical temperature $T / \varepsilon_{F}=0.03$ which applies to high$T_{c}$ superconductors (see Sec. IVD). We first point out that the $\tilde{q}$-dependence of this function is essentially restricted to the interval $\left[0,4 k_{F}\right]$.

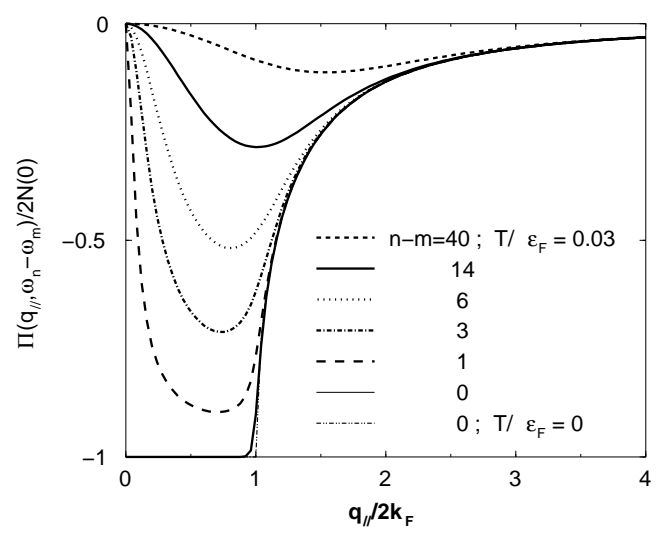

FIG. 3: Electronic polarizability in RPA as a function of $\tilde{q}=q_{\|} / 2 k_{F}$ for various values of $\omega_{n}-\omega_{m}=2(n-m) \pi T$ (from top to bottom) and a typical temperature $T / \varepsilon_{F}=0.03$. The lowest, dash-dotted curve is obtained for $T=0 \mathrm{~K}$ and remains the same for all frequencies [i.e. at $T=0, \Pi(q<$ $\left.\left.2 k_{F}, \omega_{n}-\omega_{m}\right)=-2 N(0)\right]$.

Let us now consider the temperature dependence of the polarizability. First we discuss the case $T=0$. For the real-frequency polarizability the analytical continuation gives the result derived by Stern in Ref. 24. On the other hand, in the Matsubara temperature-Green's function formalism the polarizability does not depend on frequency for $\omega<q_{\|} v_{F}$ (cf. Ref. 15a, Sec. 20.2): $\Pi\left(q, \omega_{n}\right)=-2 N(0)=-m_{b} / \pi \hbar^{2}$ (bottom dotted line of Fig. 3). For $T>0$ the polarizability vanishes at high frequencies as shown in Fig. 3] The higher the temperature, the smaller is the frequency-range over which the polarizability remains finite. Note that the shape of $\Pi\left(\tilde{q}, \omega_{n}-\omega_{m}=0\right)$ (lower solid line on the figure) is almost unaltered until very high temperatures. This can also be directly seen from the analytical expression [see appendix, Eq. (B8)].

Using the above results for the polarizability we calculate the dynamic dielectric function, Eq. (10), or rather it inverse $\epsilon^{-1}\left(\mathbf{q}, \omega_{n}-\omega_{m}\right)$ since it is this quantity that enters the vertex $\Gamma_{c}$, Eq. (5). The result is shown on Fig. 4 for the same values of parameters as in Fig. 3. We point out a few important properties of the inverse dielectric function. This function is bounded for all $\mathbf{q}$ and $\omega_{n}-\omega_{m}$. For high frequencies and/or large wave-vectors $\epsilon^{-1}\left(\mathbf{q}, \omega_{n}-\omega_{m}\right) \rightarrow 1$, meaning that the Coulomb interaction is unscreened in these cases. In fact, it can be shown 


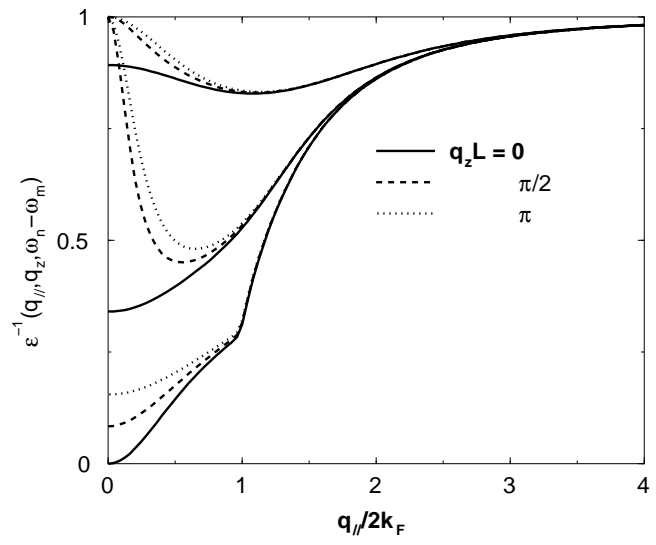

FIG. 4: Inverse dielectric function of the LEG. The solid, dashed, and dotted lines correspond to $q_{z} L=0, \pi / 2, \pi$ respectively. The three bottom curves are obtained for $\omega_{n}-\omega_{m}=0$, the three middle curves are for $n-m=10$ and the three uppermost curves are for $n-m=40$. The other parameters were given in the previous figure. Note that $\epsilon^{-1}\left(\mathbf{q} \rightarrow 0, \omega_{n}-\omega_{m}\right)$ is zero (perfect screening) only in the static limit.

that for $\hbar q v_{F} \ll \omega_{n}-\omega_{m}$ the dielectric function takes the form 17

$$
\epsilon\left(\hbar q v_{F} \ll \omega_{n}-\omega_{m}\right) \simeq \epsilon_{D}(n-m),
$$

where

$$
\epsilon_{D}(n-m) \equiv 1+\frac{\Omega_{p l}^{2}}{\left(\omega_{n}-\omega_{m}\right)^{2}} .
$$

Eq. (15) describes the dielectric function in the Drude limit. Note that latter expression is exact in the limit $\mathbf{q}=0$ and $\omega>0,17$

The result of Fig. 4 shows that for any finite frequency, the long wave-length limit takes the form $\epsilon(|\mathbf{q}| \rightarrow$ $\left.0, \omega_{n}-\omega_{m}>0\right) \rightarrow \epsilon_{D}\left(\omega_{n}-\omega_{m}\right)$. Thus, it is only in the static case $\omega_{n}-\omega_{m}=0$ that the Coulomb interaction is "perfectly" screened (exponential screening in real space $): \epsilon\left(\mathbf{q} \rightarrow 0, \omega_{n}-\omega_{m}=0\right) \approx\left[1+\kappa_{T F}^{2} /|\mathbf{q}|^{2}\right]^{-1}$. The latter limit is the so-called Thomas-Fermi screening of the Coulomb potential. For the LEG the screening length is given by $\kappa_{T F}^{2}=\pi N(0) \Omega_{p l}^{2}$. In all other cases the limit of long wave-lengths is given by the Drude limit.

The dielectric function describing the screening in layered conductors will be used in the next section to calculate the effect of the dynamically screened Coulomb interaction on $T_{c}$ in several classes of layered superconductors. We thereby use the full wave-vector, frequency and temperature dependence of $\epsilon\left(\mathbf{q}, \omega_{n}-\omega_{m}\right)$ calculated in this section.

\section{APPLICATION TO VARIOUS LAYERED SYSTEMS}

In this section we consider the phonon-plasmon mechanism and in particular the impact of the dynamic screening of the Coulomb interaction on the superconducting state of several layered systems. We discuss specific examples belonging to three classes of materials: metallochloronitrides, organics and high- $T_{c}$ superconductors. To this aim, we first rewrite Eqs. 2a 2b in a form adequate for layered conductors and convenient for calculations. We then evaluate the critical temperature $T_{c}$ of the various compounds.

\section{A. Numerical analysis}

We assume isotropy within the layers. The order parameter and the renormalization function can therefore be written as $\Delta_{n}(\mathbf{k}) \simeq \Delta_{n}\left(\mathbf{k}_{\|}=\mathbf{k}_{F}, k_{z}\right) \equiv \Delta_{n}\left(k_{z}\right)$ and $Z_{n}(\mathbf{k}) \equiv Z_{n}\left(k_{z}\right)$ (see appendix $\mathbf{C}$ ). This approximation is valid since the Cooper instability (see, e.g., Ref. 15) and, correspondingly, the pairing, occurs on the Fermi surface. As for the integrands in Eqs. 15, they depend mainly on the momentum transfer $\mathbf{q}_{\|}$. Thus, in the layered electron gas the order parameter depends on the frequency and the wave-vector perpendicular to the layers.

To perform numerical calculations with Eqs. (2a 2b we follow the standard procedure adapted to the case of layered materials. We first express the integral over $\mathbf{k}_{\|}$ in terms of an integral over energy and in-plane wavevector amplitude and carry out the former analytically (see appendix [C). We thereby reduce the equations to a form containing one-dimensional $\mathbf{k}$-space integrals. Note, however, that the dielectric function also contains an integral to be performed at each iteration of the calculation [Eqs. (1014] ]. The resulting equations take the form (appendix [C]

$$
\begin{aligned}
\phi_{n}\left(k_{z}\right) & = \\
\pi T & \sum_{m=-\infty}^{\infty} \frac{1}{N_{z}} \sum_{k_{z}^{\prime}=-\pi}^{\pi} \bar{\Gamma}\left(q_{z}, n-m\right) \frac{\phi_{m}\left(k_{z}^{\prime}\right)}{\left|\omega_{m}\left(k_{z}^{\prime}\right)\right|}, \\
\omega_{n}\left(k_{z}\right)-\omega_{n} & = \\
\pi T & \sum_{m=-\infty}^{\infty} \frac{1}{N_{z}} \sum_{k_{z}^{\prime}=-\pi}^{\pi} \bar{\Gamma}\left(q_{z}, n-m\right) \frac{\omega_{m}}{\left|\omega_{m}\right|},
\end{aligned}
$$

where $N_{z}$ are the number of $k_{z}$ points taken in the first Brillouin zone and the kernel is given by

$$
\bar{\Gamma}\left(q_{z}, n-m\right)=\lambda D(n-m)+\lambda_{c} \Gamma_{c}\left(q_{z}, n-m\right),
$$

with

$$
\begin{gathered}
\lambda \equiv \frac{N(0)}{\pi} \int_{0}^{1} \frac{\mathrm{d} \tilde{q}}{\sqrt{1-\tilde{q}^{2}}}\left|g_{\nu}(\tilde{q})\right|^{2}, \\
\Gamma_{c}\left(q_{z}, n-m\right) \equiv \\
\frac{1}{\pi} \int_{0}^{1} \frac{\mathrm{d} \tilde{q}}{\sqrt{1-\tilde{q}^{2}}} \frac{R\left(\tilde{q}, q_{z}\right) / \tilde{q}}{\epsilon\left(\tilde{q}, q_{z}, n-m\right)} .
\end{gathered}
$$

All other quantities were defined in Sec. III Two simplifications are made in the following calculations that allow to single out the effect of low-energy electronic collective 
modes on superconductivity. The first is to replace the phonon contribution to the pairing to one (or two, see below Sec. IV C characteristic phonon modes. The second is that we can set $k_{z}=\pi / L$, based on the analysis made in Sec. III which shows that this wave-vector gives the largest contribution to the pairing. Consequently, the order parameter and the renormalization function taken at the zone boundary along $k_{z}$ are function of frequency $\omega_{n}$ only. We emphasize, however, that these two simplifications are not affecting the main results presented below. Rather, they would lead to smaller coupling constants necessary to reach a specific critical temperature $T_{c}$. For example, we expect that taking into account all plasmon acoustic plasmon branches would lead to higher critical temperatures (or correspondingly to smaller Coulomb coupling constants for a given $T_{c}$ ) since they contribute to the attractive pairing interaction, though with lesser weight, 13.14

As discussed in Sec. IIIA the excitation spectrum of a layered electron gas (see Fig. 2) allows us to divide the $\left(q_{\|}, \omega\right)$-space into two main regions. The first (region I) contains the additional collective excitations discussed here and this region should be considered exactly in the equations above. On the other hand, region II contains no such additional plasmon features and the equations have a form analog to the $3 \mathrm{D}$ case in this area. Therefore, we make a further step by dividing the Coulomb part, Eq. 19b, into two parts corresponding to the two regions of Fig. 2

$$
\begin{aligned}
\Gamma_{c}\left(q_{z}, n-m\right) & =\Gamma_{c}^{\mathrm{I}}\left(q_{z}, n-m\right)+\Gamma_{c}^{\mathrm{II}}\left(q_{z}, n-m\right)= \\
& \frac{1}{\pi}\left\{\int_{0}^{\tilde{q}_{c}}+\int_{\tilde{q}_{c}}^{1}\right\} \frac{\mathrm{d} \tilde{\mathrm{q}}}{\sqrt{1-\tilde{q}^{2}}} \frac{R\left(\tilde{q}, q_{z}\right) / \tilde{q}}{\epsilon\left(\tilde{q}, q_{z}, n-m\right)},
\end{aligned}
$$

where $q_{c}=\min \left\{2 k_{F},\left|\omega_{n}-\omega_{m}\right| / \hbar v_{F}\right\}$ and $\tilde{q}_{c} \equiv q_{c} / 2 k_{F}$. The part $\Gamma_{c}^{\mathrm{I}}$ will be considered exactly, in particular with respect to the frequency dependence that has the distinctive features of layered conductors. The part $\Gamma_{c}^{\mathrm{II}}$, on the other hand, is reduced to an effective constant Coulomb pseudopotential $\mu^{\star} \theta\left(\omega_{n}-\Omega_{c}\right)$ with a standard cutoff given by $\Omega_{c} \simeq 10 \times \Omega(\Omega$ is the characteristic phonon energy). This treatment of region II calls for a comment. As was mentioned earlier we consider usual phononmediated superconductivity (Eliashberg equations) as a starting point of our analysis. Accordingly, the electronphonon coupling constant and the static term $\mu^{\star}$ are treated in the conventional way as parameters to be determined from experimental data. Thus, the static term $\mu^{\star}$ is here a phenomenological parameter. We focus on the term describing the contribution of the dynamic screening. This part (present in region I) will be evaluated explicitely for different systems with the use of normal-state parameters (as, e.g., $v_{F}$ or $\epsilon_{M}$; see below). Note that for the pure plasmon mechanism both static and dynamic terms were calculated in Ref. 7 (in 3D). This step was crucial since its value was directly related to the criterion for the appearence of superconductivity. For the phonon-plasmon mechanism, on the other hand, we assume that the phonons are sufficient for the occurence of the superconducting state, which allows to use the conventional approach for the static term. Naturally, it would be of great interest to calculate the static term. Such a full self-consistent calculation including also real band structures will be carried out elsewhere.

As shown in appendix $\mathrm{C}$ (see also Ref. 25), the above equations can be mapped onto an eigenvalue problem written in tensorial form

$$
\underline{\underline{\mathbf{K}}} \underline{\Phi}=\eta \underline{\Phi}
$$

where $(\underline{\Phi})_{n, n_{z}}=\Phi_{n}\left(n_{z}\right) \equiv \Delta_{n}\left(n_{z}\right) / \sqrt{2 n+1}$ and $\underline{\underline{\mathbf{K}}}$ is given by Eq. (C3) in appendix C Eq. (21) is written explicitely in Eq. C4. Note that an artificial eigenvalue $\eta$ has been introduced in Eq. 21). $T_{c}$ is reached when $\eta$ is one. Since all eigenvalues satisfy the inequality $\eta \leq 1$ we only need studying the highest of them. Furthermore, the solution of these equations give also the renormalized order parameter $\Phi_{n}$ near $T_{c}$. We can thus analyse how this function is affected by the contribution of low-energy plasmons to the pairing interaction. A typical function $\Phi_{n}$ is shown in Fig. [5] and will be discussed in the next section. Using the eigenvalue equation (21) [or (C4) in appendix $[$ we apply the theory to various layered superconductors and calculate their $T_{c}$.

\section{B. Intercalated metal halide nitrides}

The first class of materials we consider is the family of layered metal-intercalated halide nitrides. We give special attention to this family because low-energy plasmons not only contribute to the pairing but, in fact, play the key role for the superconducting state, as we show below. We believe that this is the first observed system where the superconducting state of the electrons is essentially self-supported, that is, where the pairing is provided by collective excitations of the same carriers as those forming pairs.

This family of novel superconductors has been discovered recently and studied in detail in Refs. 182627.28 , 29303132,33 34 35 36. As is known (see, e.g., Ref. 26) the intercalation of alkali atoms and organic molecules into the parent compound ( $\mathrm{Zr}, \mathrm{Hf}) \mathrm{NCl}$ leads to a superconductor with rather high critical temperature $\left(T_{c} \sim\right.$ 25K). Based on experimenta118.26.27.28.29.30.31.32.33 studies and band structure calculations ${ }^{35}$ it was concluded that electron-phonon mediated pairing is insufficient to explain the observed $T_{c}$, since the electron-phonon coupling constant appears to be too small. Note also that a small nitrogen isotope effect of $T_{c}$ has been observed ${ }^{32}$ In addition, the compounds do not contain any magnetic ions and no sign of magnetism has been found in band structure calculations. This excludes a magnetic pairing mechanism. Finally, normal-state properties of the materials studied intensively in Refs. 18 35.36 can be described by Fermi liquid theory, so that there is no evidence for the presence of strong correlations. 
We apply our approach to this novel layered system. We note from Eqs. (91720), that the evaluation of $T_{c}$ for a specific compound needs the knowledge of following parameters: the interlayer distance $L$, the band mass $m_{b}$ and Fermi velocity $v_{F}$, and the dielectric constant of the spacers $\epsilon_{M}$. In addition, the evaluation of the phonon contribution to the pairing requires the knowledge of the characteristic phonon frequency $\Omega$, the electron-phonon coupling constant $\lambda$ and the Coulomb pseudopotential $\mu^{\star}$.

Specifically, we consider $\mathrm{Li}_{0.48}(\mathrm{THF})_{y} \mathrm{HfNCl}$ (THF=tetrahydrofurane) as an example, since the largest amount of information necessary for the determination of $T_{c}$ is available for this material, both from experiment and band structure calculations. According to Refs. 183034 the interlayer distance $L$ and the characteristic phonon frequency $\Omega$ are equal to: $L=18.72 \AA$ and $\Omega=60 \mathrm{meV}$. The values of the band mass and Fermi energy have been evaluated from band structure calculations, Ref. 35. Accordingly, $m_{b}=0.6 m_{e}$, where $m_{e}$ is the free electron mass and $\varepsilon_{F} \simeq 1 \mathrm{eV}$. For $\epsilon_{M}$ we have chosen the reasonable value $\epsilon_{M}=1.75$. It follows that $\lambda_{c} \simeq 0.8$ and, correspondingly, using Eq. (9), $r_{s} \simeq 2$ (i.e. close to the high-density limit). The value of the electron-phonon coupling constant can be estimated from the knowledge of the electron specific heat constant $\gamma$ and the band density of states $N(0)$. Indeed, the electron-phonon interaction renormalizes $\gamma$ as $\gamma=\gamma_{b}(1+\lambda)$, where $\gamma_{b}=2 \pi^{2} N(0) / 3$ is the free electron Sommerfeld constant. The value of $\gamma$ was estimated in Ref. 18 to be $\gamma \simeq 1.1 \mathrm{~mJ} / \mathrm{molK}^{2}$, whereas band structure calculations ${ }^{35}$ give $N(0) \simeq 0.74 \mathrm{eV}^{-1}$. Thus, $\lambda \simeq 0.25$. Setting $\mu^{\star}=0.1$ and using Eqs. (1720), we obtain $T_{c} \simeq 24.5 \mathrm{~K}$. The calculated $T_{c}$ is very close to the observed value $T_{c}^{e x p}=25.5 \mathrm{~K}$. The essential point to note is that in absence of the plasmon contribution we obtain $T_{c}^{\text {phonon }} \ll 1 \mathrm{~K}(!)$. This demonstrates that, indeed, the low-energy plasmon contribution plays a key role for superconductivity in metallochloronitrides.

It would be of great interest to carry out specific tunneling (cf. Ref. 9) and optical measurements on this material. We expect that tunneling experiments, similarly to heat capacity data (see above), will provide the value $\lambda \simeq 0.25$, and optical measurements will lead to $\epsilon_{M} \simeq 1.75$.

As mentioned earlier, by solving Eq. (17) we not only obtain $T_{c}$, but we also get the superconducting order parameter $\Phi_{n}=\Delta_{n} / \sqrt{2 n+1}$. It is interesting to see how $\Phi_{n}$ is affected by the additional pairing arising from the presence of acoustic plasmons (Fig. 5). Note that the qualitative form of the order parameter is the same for all classes of materials discussed in the present paper. In the absence of the plasmon contribution, the order parameter is a rapidly decreasing monotonic function of the Matsubara frequency (dotted line of Fig. 5 ). The effect of plasmons reveals itself as an additional "step" in $\Phi_{n}$ at

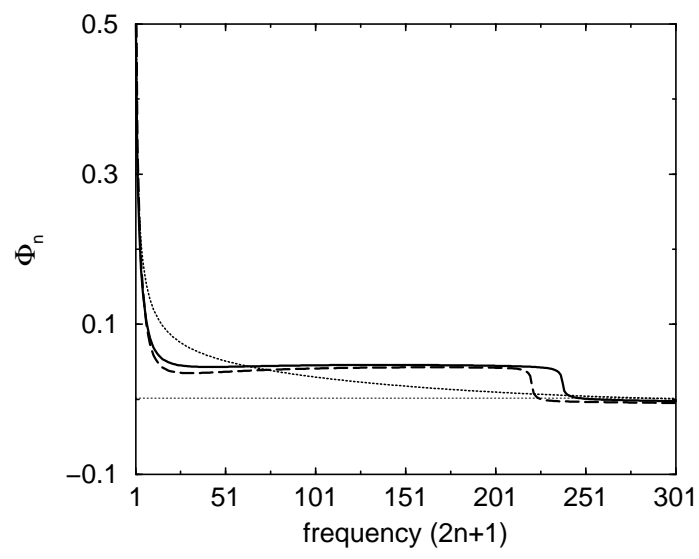

FIG. 5: Normalized order parameter $\Phi_{n}=\Delta_{n} / \sqrt{2 n+1}$ as a function of Matsubara frequency $\omega_{n}=(2 n+1) \pi T$. The solid and dashed lines obtained for $\lambda=1$ and 1.5 respectively, represent the order parameter in the presence of the plasmon contribution. The dotted line was obtained in absence of the plasmon contribution $\left(\lambda_{c}=0, \lambda=1\right)$. Note the presence of an additional structure (step) when including the pairing due to low-energy plasmons (solid and dashed lines). $\mu^{\star}=0.1$.

intermediate frequencies, as examplified by the solid and dashed lines on Fig. [5 It is this positive part of the order parameter due to the pairing induced by the low-energy collective modes that is responsible for the enhancement of the value of $T_{c}$. We observe that the order parameter remains positive over a frequency range also determined by the value of $\lambda$ and $\mu^{\star}$. Whereas the frequency range over which $\Phi_{n}$ remains positive shrinks with increasing $\lambda$ (compare solid and dashed lines on Fig. 5) it increases with increasing $\mu^{\star}$ (not shown on the figure). This apparently counter-intuitive behaviour is easily understood by the fact that an order parameter extending to higher frequencies will pick up more and more repulsive components of the pairing interaction. The "shorter" the step in frequency of the plasmon-induced structure, the smaller is the repulsive contribution of the effective interaction kernel and, consequently, the higher is $T_{c}$.

Concluding this section, we emphasize that the dynamic screening of the Coulomb interaction (the contribution from low-energy electronic collective modes) is essential for the understanding of the superconducting state in intercalated layered metal halide nitrides.

\section{Layered organic superconductors}

Organic superconductors were predicted in Ref. 37 and discovered in Ref. 38. In this section we apply the theory to the class of layered organic superconductors (see, e.g., Ref. 39 40,4143 and references therein). As an example, we focus on $\kappa-(\mathrm{ET})_{2} \mathrm{Cu}(\mathrm{NCS})_{2}(\mathrm{ET}=\mathrm{BEDT}-\mathrm{TTF}$ is a short notation for bisethylenedithiotetrathiofuvalene). The basic structural building blocks of these materials are large, elongated ET planar molecules stretching along the 
$c$-axis forming the conducting layer $\underline{\underline{44}}$ These thick conducting layers are separated by thin insulating spacers of planar NCS-molecules extending in the $a b$-plane. The NCS counter-ions take one charge per two ET-molecules leaving the ET HOMO partially unfilled. The HOMO are $\pi$-holes delocalized over the large organic molecule and form the hole conduction bands.

Layered organic conductors have highly anisotropic transport properties. Typically, the ratio of in- to outof-plane conductivity is at least of the order $\sigma_{\|} / \sigma_{\perp} \sim$ $10^{4}, 42.45$ Band structure calculations 46 confirm the presence of quasi-two-dimensional bands. We emphasize once more, however, that only electronic transport properties are quasi-2D. As discussed in the previous sections, the Coulomb interaction is important in all three dimensions. In particular, incomplete screening between layers implies that carriers from differents layers interact with each other, leading to the low-energy electronic collective modes discussed here. As we show in the following, this aspect is important for understanding the relatively high value of the critical temperatures observed in these materials.

Superconductivity has been observed for temperatures $T<T_{c} \simeq 10.4 \mathrm{~K}$. Recent studies have shown the importance of electron-phonon interaction for the pairing mechanism. 48,49,50,51.52,53 For example, isotope effect studies of the superconducting $T_{c}$ by isotope substitution of $\mathrm{C}$ and $\mathrm{S}$ atoms on the ET-molecules have singled out the effect of intramolecular vibrations for the superconducting pairing 48.49 A shift of phonon frequency caused by the superconducting transition has also been observed with inelastic neutron scattering $\underline{50}$ This shift indicates that the coupling to intermolecular acoustic phonons contributes to superconductivity. Further work supporting the importance of electron-phonon interaction for superconductivity are given in Refs. 51,52,53. Therefore, it is interesting to apply our phonon-plasmon model to this class of materials and study the effect of acoustic plasmons on the superconducting $T_{c}$.

As it appears that both inter- and intramolecular vibrational modes are of importance to superconductivity we modelize the phonon kernel in Eq. (3) by a two-peak function

$$
\begin{aligned}
& \Gamma_{p h}\left(\omega_{n}-\omega_{m}\right)= \\
& \quad \lambda\left[w_{1} \frac{\Omega_{1}^{2}}{\left(\omega_{n}-\omega_{m}\right)^{2}+\Omega_{1}^{2}}+w_{2} \frac{\Omega_{2}^{2}}{\left(\omega_{n}-\omega_{m}\right)^{2}+\Omega_{2}^{2}}\right] .
\end{aligned}
$$

The lower frequency mode $\Omega_{1}=5 \mathrm{meV}$ corresponds to libration and intermolecular modes $\stackrel{42.50}{ }$ The higher frequency peak is located at the frequency $\Omega_{2}$, and was calculated for the ET-intramolecular vibrations: $\Omega_{2}=$ $10 \mathrm{meV} \stackrel{52.54}{ }$ Given the number of modes present near each peak and their possible coupling to the electrons, we set $w_{1}=0.75$ and $w_{2}=0.25$. The coupling constants to each set of modes is then defined as $\lambda_{j}=\lambda w_{j}$ $(j=1,2)$.

To calculate the value of $T_{c}$, we need to know the value of the band mass $m_{b}$, the interlayer distance $L$, the Fermi energy $\varepsilon_{F}$ and the dielectric constant of the spacers $\epsilon_{M}$ (normal state parameters), as well as the value of the electron-phonon coupling constant $\lambda$ and the Coulomb pseudopotential $\mu^{\star}$. From band structure calculations we have $m_{b}=1.72 m_{e}{ }^{46}$ The structure determination gives $L=16.2 \AA \stackrel{44}{\underline{4}}$ The average value of the Fermi wavevector obtained from Shubnikov-de Haas measurements is $k_{F} \simeq 2.6 \times 10^{7} \mathrm{~cm}^{-1}$ (Ref. 45). Inserting these values in $\varepsilon_{F} \equiv \hbar^{2} k_{F}^{2} / 2 m_{b}$ we obtain $\varepsilon_{F} \simeq 0.17 \mathrm{eV}$. Note that this is exactly the value obtained from band structure

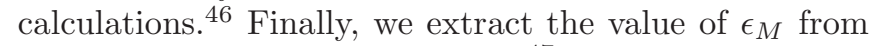
optical reflectance measurements $\stackrel{47}{=}$ Using Eqs. (D1 D2) and the data of Ugawa et al $\underline{47}$ we obtain $\epsilon_{M}=6.5$ (appendix (D). Note that the ionic screening of the Coulomb interaction is more efficient in organics than in metallochloronitrides (previous section). One reason for this difference is given by the fact that in organics the thick conducting slabs are made of large molecules, whereas in the metallochloronitrides conducting sheets are thin and made of covalently bond atoms. The polarizability of the molecules implies better ionic screening of the Coulomb interaction and, therefore, a larger value of $\epsilon_{M}$. These parameters lead to $\lambda_{c} \simeq 0.9$ and thus $r_{s} \simeq 2.5$.

The exact value of the electron-phonon coupling constant $\lambda$ is unknown at present. Estimates range $\lambda$ between 0.5 and $1.5 \underline{42.52 .54}$ Consequently, we present results for this range of values in Fig. [6] (we have chosen $\mu^{\star}=0.1$ and the cutoff at $\left.\Omega_{c}=10 \times \Omega_{2}=0.1 \mathrm{eV}\right)$. Using these parameters we calculate $T_{c}$ from Eqs. (17)18) (see Fig. [6).

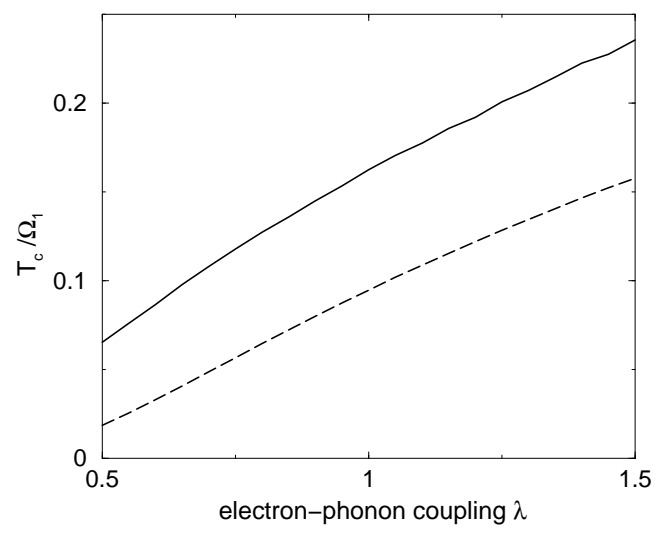

FIG. 6: $T_{c}(\lambda)$ where $\lambda$ is defined in Eq. (22). $T_{c}$ is normalized to the lowest phonon energy $\Omega_{1}$. The upper (lower) curve is obtained in the presence (absence) of the acoustic plasmon contribution. $\mu^{\star}=0.1$.

The result shows that the increase of $T_{c}$ in the presence of low-energy electronic collective modes is substantial. We can quantify this enhancement of $T_{c}$ for the specific example studied, the $\kappa-(\mathrm{ET})_{2} \mathrm{Cu}(\mathrm{NCS})_{2}$ compound. According to our calculation (see Fig. (6) the experimentally observed value $T_{c}=10.4 \mathrm{~K}$ is obtained for $\lambda \simeq 1$, implying a coupling to the low- and high-energy phonon 
modes of $\lambda_{1}=0.83$ and $\lambda_{2}=0.28$, respectively. Thus, $\kappa-(\mathrm{ET})_{2} \mathrm{Cu}(\mathrm{NCS})_{2}$ is an intermediate coupling superconductor. In the absence of acoustic plasmons we obtain $T_{c}^{\text {phonon }}=6.3 \mathrm{~K}$ for this $\lambda$. Thus, in the present case $40 \%$ of the value of $T_{c}$ is due to the pairing of electrons via the exchange of acoustic plasmons. These calculations lead us to conclude that the contribution of low-energy electronic collective modes to the pairing is significant in organic superconductors (though not dominant as in the case of metallohalidenitrides, Sec. IVB).

\section{High-temperature oxides}

In this section we discuss superconductivity in the cuprates within our phonon-plasmon model. We analyze one specific material, $\mathrm{La}_{1.85} \mathrm{Sr}_{0.15} \mathrm{CuO}_{4}$, for which most parameters have been determined. The normal state parameters are: the interlayer distance $L=6.5 \AA$, the Fermi wave-vector $k_{F}=3.5 \times 10^{7} \mathrm{~cm}^{-1}$, the characteristic phonon frequency $\Omega \simeq 15 \mathrm{meV}$, the dielectric constant $\epsilon_{M} \simeq 5-102^{23.55}$ Therefore, $\lambda_{c} \simeq 1$ and $r_{s} \simeq 2.8$. The effective mass $m^{\star}$ and electron-phonon coupling constant $\lambda$ were determined by Wolf and one of the authors from heat capacity measurements ${ }^{56.57}$ The obtained values are $\lambda=2$ and $m^{\star} \approx 5 m_{b}$. From the relation $m^{\star}=(1+\lambda) m_{b}$ we then obtain $m_{b} \simeq 1.7$. Finally, the Coulomb pseudopotential is taken to be $\mu^{\star} \simeq 0.1$.

The solution of Eqs. 17a 17b 18 with use of the aforementioned parameters lead to $T_{c}=36.5 \mathrm{~K}$ which is close to the experimental value $T_{c}^{e x p} \simeq 38 \mathrm{~K}$. It is essential to note that in the absence of the screened Coulomb interaction we would obtain $T_{c}^{p h}=30 \mathrm{~K}$. Thus, about $20 \%$ of the observed value of $T_{c}$ is due to "acoustic" plasmons. For thin films the stiffness of the lattice usually increases, leading to a higher value of the characteristic phonon frequency $\Omega$. Assuming $\Omega=20 \mathrm{meV}$, we obtain $T_{c}=49 \mathrm{~K}$ which is close to the experimental value $T_{c}^{\exp }=45 \mathrm{~K}$ observed, e.g. in Ref. 57. This value is indeed higher than the one of bulk samples. Interestingly, we obtain $T_{c}^{p h}=40 \mathrm{~K}$, so that the increase of $T_{c}$ induced by "acoustic" plasmons is again of order of $20 \%$.

Thus, the dynamically screened interlayer Coulomb interaction is important for superconductivity in the cuprates. Note that a proper account of the Coulomb interaction screening is not only of importance for superconductivity in these materials, but also for a proper description of normal state properties such as lattice dynamics. 22,23

\section{DISCUSSION AND CONCLUSIONS}

The purpose of the present article was to study the impact of layering on superocnductivity. Particular emphasis was set on the dynamically screened Coulomb interaction. Layered materials have distinctive low-energy electronic collective excitations that provide exchange bosons for the pairing between electrons. We have shown that these acoustic plasmons lead to an enhancement of the superconducting $T_{c}$. We have applied the theory to three classes of layered superconductors: alkali-intercalated halide nitrides, organic and high-temperature superconductors.

Within our phonon-plasmon model we observe an increasing influence of the electronic pairing mechanism for the three classes of layered superconductors considered. In metal-intercalated halide nitrides the contribution arising from low-energy electronic collective modes is dominant. These materials are thus unique in the sense that an electronic pairing mechanism is at the origin of superconductivity: the exchange bosons are made of the same particles (the electrons) than those who bind into pairs below $T_{c}$. In the case of organic layered materials, the electronic and phononic energies, as well as the structure of the conducting layers and insulating spacers, leads to a situation where the contribution of phonons and acoustic plasmons is of the same order. Finally, in the case of high-temperature superconductors, the contribution of low-energy plasmons is significant but not dominant. Within our model the phonon contribution is still largest.

There are other classes of layered superconductors that have not been considered in the present article. Among them, the most prominent is that of dichalcogenides. We believe that some experimental observations 58 are related to the phenomenon discussed in this article. However, we also note that many of the systems belonging to this class of materials exhibit charge density wave instabilities. This both obscures and changes the contribution of acoustic plasmons to superconductivity and will be discussed elsewhere.

Another interesting system is the $\mathrm{CoO}_{2}$-based layered compound studied recently in Ref. 1 . We point out that the system becomes superconducting only for relatively large interlayer distance. This is consistent with the present theory and the material deserves further study.

An essential conclusion of the present work is that the physics of layered (super)conductors cannot be reduced to the study of one conducting layer (or the layers belonging to one unit cell as in some high-temperature superconductors). Such simplification relies on the observation of "quasi-two-dimensional" transport. However, it misses to account for the screening properties of the electronelectron Coulomb interaction (and of the electron-phonon interaction as well; see Refs. 22 59). As we discussed in the paper, the screening is very different in layered materials as compared to $2 \mathrm{D}$ and $3 \mathrm{D}$ isotropic metals. We believe that the particular screening properties are essential for the behaviour of layered (super)conductors. How large the effect of screened Coulomb interaction is, depends very much on the specific features of the material. For example, the covalency within the conducting layers and the structure of the spacers, the presence of van der Waals gaps will determine its contribution both to normal and superconducting state properties. Therefore, 
the study of screening properties in layered conductors is a promising direction to better understand the similarities and differences between different classes of materials and serve as a bridge in the study of properties of $2 \mathrm{D}$ and $3 \mathrm{D}$ systems.

\section{ACKNOWLEDGEMENTS}

We thank I. Bozovic, D. van der Marel and S.A. Wolf for fruitful discussions. H.M. thanks P. Reineker and the Institute of Theoretical Physics of Ulm University for discussions and hospitality. A.B. thanks the Max Planck Institute for the Physics of Complex Systems, Dresden, for hospitality.

\section{APPENDIX A: COULOMB POTENTIAL FOR LAYERED SYSTEMS}

Using cylindrical coordinates $\mathbf{r}=\left(\mathbf{r}_{\|}, z\right)$ (where $z$ is perpendicular to the layers) the Fourier transform of the $3 \mathrm{D}$ Coulomb potential $V_{c}(\mathbf{r})=e^{2} / \epsilon_{M}|\mathbf{r}|$ is given in layered structures by 21

$$
\begin{aligned}
V_{c}(\mathbf{q}) & =\frac{1}{N_{z}} \sum_{n} e^{-\mathrm{i} q_{z} n L} \int \mathrm{d} \mathbf{r}_{\|} e^{-\mathrm{i} \mathbf{q}_{\|} \mathbf{r}_{\|}} \frac{e^{2} \epsilon_{M}^{-1}}{\mathbf{r}_{\|}^{2}+(n L)^{2}} \\
& =\frac{1}{N_{z}} \sum_{n} e^{-\mathrm{i} q_{z} n L} \frac{2 \pi e^{2}}{\epsilon_{M} q_{\|}} e^{-q_{\|} n L},
\end{aligned}
$$

where we have taken into account the fact that the charges are located in the conducting sheets, and thus $z=n L$ where $L$ is the interlayer spacing and $n$ indexes the layers. Note that the second line of Eq. (A1) shows how the Coulomb interaction is exponentially decaying (in real space) along the direction perpendicular to the layers, the decay being determined by $q_{\|} L$. Performing the sum in Eq. (A1) we obtain Eq. (6).

Note that the detailed structure of the spacers separating the conducting sheets is not considered in the present model. We thus have included the screening resulting from polarization effect of the spacers via the dielectric constant $\epsilon_{M}$. The dielectric function in the denominator of Eq. (5) thus accounts for the screening induced by the charge-carriers of the conduction bands only.

\section{APPENDIX B: POLARIZABILITY}

The RPA polarizability of a single conducting sheet, Eq. (14), is written in polar coordinates $\mathbf{k}_{\|}=(k, \varphi)$

$$
\begin{array}{r}
\Pi\left(\mathbf{q}_{\|}, i \omega_{n}\right)=\frac{2}{(2 \pi)^{2}} \int_{0}^{\infty} \mathrm{d} k k f_{k} I_{\varphi}\left(k, \mathbf{q}_{\|}, \omega_{n}\right),(\mathrm{B} 1) \\
I_{\varphi}\left(k, \mathbf{q}_{\|}, i \omega_{n}\right)= \\
\int_{0}^{2 \pi} \mathrm{d} \varphi\left\{\frac{1}{i \omega_{n}+\xi_{\mathbf{k}_{\|}}+\xi_{\mathbf{k}_{\|}+\mathbf{q}_{\|}}}-\frac{1}{i \omega_{n}+\xi_{\mathbf{k}_{\|}}-\xi_{\mathbf{k}_{\|}+\mathbf{q}_{\|}}}\right\} .
\end{array}
$$

For $T=0$ the integral over $\mathbf{k}_{\|}$can be calculated analytically, leading to the result first derived in Ref. 24. At finite temperature there is no simple analytical form. However, to reduce the amount of numerical work in solving Eqs. 17a 17b for $T_{c}$, we calculate the angle integral analytically. Using the transformation $z=\exp (i \varphi)$ we integrate $I_{\varphi}$ over $\mathrm{z}$ in the complex plane to obtain

$$
I_{\varphi}=-\frac{\sqrt{2} \pi}{q_{\|}} \frac{u_{+}}{W}\left[\delta(\tilde{k})+\theta_{1}-\theta_{2}\right]
$$

with $u_{ \pm}=\sqrt{A \pm W}, W=\sqrt{A^{2}+B^{2}}, A=4 \tilde{k}^{2} \tilde{q}^{2}\left(\zeta_{1}^{2}-\right.$ $\left.\zeta_{2}^{2}-1\right), B=4 \tilde{k}^{2} \tilde{q}^{2} \zeta_{1} \zeta_{2}$, and

$$
\zeta=\zeta_{1}+i \zeta_{2} \equiv \frac{\tilde{q}}{2 \tilde{k}}+i \frac{\tilde{\omega}_{n}}{4 \tilde{k} \tilde{q}}
$$

As in the main text we normalize all wave-vectors to $\tilde{X} \equiv X / 2 k_{F}, X=q, k \cdot \theta_{j}(j=1,2)$ are defined in terms of Heavyside functions as $\theta_{j}=\theta\left(1-\left|z_{j}\right|^{2}\right), z_{j}=x_{j}+i y_{j}$ with

$$
\begin{gathered}
x_{1}=\left|u_{+}\right|-\zeta_{1}, \quad y_{1}=\left|u_{-}\right|-\zeta_{2}, \\
x_{2}=-\left|u_{+}\right|-\zeta_{1}, \quad y_{2}=-\left|u_{-}\right|-\zeta_{2},
\end{gathered}
$$

for $B \geq 0$ whereas $y_{1}$ and $y_{2}$ are interchanged for $B<0$. Inserting this expression into Eq. (B1) above, we obtain the following compact form for the polarizability

$$
\left.\Pi\left(\tilde{q}, \omega_{n}\right)=-N(0) \frac{\sqrt{2}}{\tilde{q}^{2}} \int_{0}^{\infty} \mathrm{d} A f_{A} \frac{\partial u_{+}}{\partial A}\left[\delta(\tilde{k})+\theta_{1}-\theta \mathrm{B}\right] 7 .\right)
$$

This expression of the polarizability has been used to calculate the dielectric function that appears in Eqs. (17a 17b and is depicted in Fig. 3 Note that

$$
\lim _{q \rightarrow 0} \Pi\left(q, \omega_{n}-\omega_{m}\right)=-2 N(0) f_{\mathbf{k}_{\mathbf{F}}} \delta_{\omega_{n}, \omega_{m}}
$$

\section{APPENDIX C: EQUATIONS FOR THE ORDER PARAMETER AND THE RENORMALIZATION FUNCTION}

We start with Eq. (2) and, as mentioned in Sec. IVA assume isotropy of the bands within the planes. Thus, $\Delta$ and $Z$ depend only on the norm of $\mathbf{k}_{\|}$(and on $k_{z}$ ). 
In this case, it is possible to calculate one of the integrals over $\mathbf{k}^{\prime} \|$ analytically. To this aim, we transform the $2 \mathrm{D}$ in-plane integration in a way analogous to the 3D cases generally studied, namely introducing polar coordinates $\mathrm{d}^{2} \mathbf{k}=\mathrm{k}^{\prime} \mathrm{dk}^{\prime} \mathrm{d} \varphi$. With $k^{\prime} \mathrm{dk}^{\prime}=2 \pi \mathrm{N}(0) \mathrm{d} \xi$ and $q^{2}=\left|\mathbf{k}^{\prime}-\mathbf{k}\right|^{2}=k^{2}+k^{\prime 2}-2 k^{\prime} k \cos (\varphi)$ the integral over $\mathbf{k}_{\|}$ is transformed into an integral over energy $\xi$ and angle $\varphi$. Using the fact that the resulting energy integrand of Eq. (2) is falling off as $\xi^{-2}$, the main contribution to this integral will come from $\xi / \varepsilon_{F} \ll 1$ and we obtain

$$
\mathrm{d}^{2} \mathbf{k}^{\prime}=2 \pi N(0) \mathrm{d} \xi \mathrm{d} \varphi \simeq 4 \pi \mathrm{N}(0) \mathrm{d} \xi \frac{\mathrm{d} \tilde{\mathrm{q}}}{\sqrt{1-\tilde{\mathrm{q}}^{2}}},
$$

with $\tilde{q}<1$. The energy integral can then easily be performed. Gathering the different terms and assuming that the electron-phonon coupling function $g_{\nu}(\mathbf{q})=g_{\nu}\left(q_{\|}\right)$, in order to define $\lambda$ and $\Gamma_{c}$ as in Eq. (19), we obtain Eq. (17). The latter equations have been obtained by discretising $k_{z}=-\pi / L+2 \pi n_{z} / N_{z} L$ with $n_{z}=1, \ldots, N_{z}$. Note, that the angle-integration can in principle be performed exactly, without need of the approximation, Eq. (C1). However, the difference with the present method is minimal and we use the approximation above for simplicity.

To perform the numerical calculation we cast Eq. (2) or (17) into a matrix form. We first transform the summation over $m=\cdots-1,0,1, \ldots$ to a sum over non-negative $m$ only. Equation (17) then takes the form

$$
\begin{aligned}
\Delta_{n}\left(k_{z}\right) Z_{n}\left(k_{z}\right) & =\pi T \sum_{m \geq 0} \frac{1}{N_{z}} \sum_{k_{z}^{\prime}=-\pi}^{\pi}\left\{\bar{\Gamma}\left(q_{z}, n-m\right)+\bar{\Gamma}\left(q_{z}, n+m+1\right)\right\} \frac{\Delta_{m}\left(k_{z}^{\prime}\right)}{\left|\omega_{m}\right|} \\
Z_{n}\left(k_{z}\right) & =1+\pi \frac{T}{\omega_{n}} \sum_{m=0}^{2 n} \frac{1}{N_{z}} \sum_{k_{z}^{\prime}=-\pi}^{\pi} \bar{\Gamma}^{\prime}\left(q_{z}, n-m\right) .
\end{aligned}
$$

The second equation has been simplified further, reducing the sum over $m$ to the range $[0,2 n]$. The kernel $\bar{\Gamma}^{\prime}$ in Eq. (C2b) now only contains frequency-dependent terms. All frequency-independent terms vanished in the folding of the summation over $m$.

Inserting Eq. C2b into C2a defining $\Phi_{n}\left(k_{z}\right)=$ $\Delta_{n}\left(k_{z}\right) / \sqrt{2 n+1}$ and

$$
K_{n m}\left(q_{z}=k_{z}^{\prime}-k_{z}\right)=\frac{1}{\sqrt{(2 n+1)(2 m+1)}}\left\{\bar{\Gamma}\left(q_{z}, n-m\right)+\bar{\Gamma}\left(q_{z}, n+m+1\right)-\delta_{n, m} \sum_{p=0}^{2 n} \bar{\Gamma}^{\prime}\left(q_{z}, n-p\right),\right\}
$$

we finally condense Eq. (C2) to the matrix form $\left(q_{z}=\right.$ $\left.k_{z}^{\prime}-k_{z}\right)$

$$
\sum_{m \geq 0} \frac{1}{N_{z}} \sum_{n_{z}^{\prime}=1}^{N_{z}} K_{n m}\left(\left|n_{z}^{\prime}-n_{z}\right|\right) \Phi_{m}\left(n_{z}^{\prime}\right)=\frac{}{\eta \Phi_{n}\left(n_{z}\right),}
$$

$n_{z}=1, \ldots, N_{z}$. This is the explicit form of Eq. (21). Note that the kernel $K_{n m}\left(q_{z}\right)$ depends on $n$ and $m$ separately and not only on $n-m$. Furthermore, the kernel is even in $q_{z}, K_{n m}\left(q_{z}\right)=K_{n m}\left(\left|q_{z}\right|\right)$. We have introduced the artificial eigenvalue $\eta$ to map the problem onto an eigenvalue equation. $T_{c}$ is obtained when $\eta=1$.

\section{APPENDIX D: DIELECTRIC CONSTANT OF THE SPACERS $\epsilon_{M}$}

The dielectric constant of the spacers $\epsilon_{M}$ can be extracted from infrared or reflectivity data. We parametrize the dielectric function obtained in these experiments by the Drude-Lorentz model

$$
\epsilon(\omega)=\epsilon_{\infty}+\sum_{j} \frac{S_{j} \omega_{j}^{2}}{\omega_{j}^{2}-\omega^{2}-i \omega \Gamma_{j}}+\epsilon_{\mathrm{fc}},
$$

where $\epsilon_{\mathrm{fc}}$ is the free carrier contribution to the dielectric constant. The dielectric constant for the spacers is then defined as

$$
\epsilon_{M}=\epsilon(\omega=0)-\epsilon_{\mathrm{fc}}=\epsilon_{\infty}+\sum_{j} S_{j} .
$$


For the determination of the dielectric constant of the organic material $\kappa-(\mathrm{ET})_{2} \mathrm{Cu}(\mathrm{NCS})_{2}$ we use Ugawa et al.'s reflectivity measurements. ${ }^{47}$ Their parametrization gives $\epsilon_{\infty}=3.2$ and

$$
\begin{array}{lll}
\omega_{1}=0.16 & \omega_{2}=0.28 & \omega_{3}=0.47 \mathrm{eV} \\
\Omega_{p 1}=0.093 & \Omega_{p 2}=0.7 & \Omega_{p 3}=0.44 \mathrm{eV}
\end{array}
$$

With the correspondance $\Omega_{p j}^{2} \equiv S_{j} \omega_{j}^{2}$ we have $S_{1} \simeq$ $0.762, S_{2} \simeq 1.581, S_{3} \simeq 0.968$. From these data and Eq. (D2) it follows that

$$
\epsilon_{M}=6.5
$$

1 K. Takada, H. Sakurai, E. Takayama-Muromachi, F. Izumi, R.A. Dilanian, and T. Sasaki, Nature 422, 53 (2003).

2 L.A. Klinkova, M. Uchida, Y. Matsui, V.I. Nikolaichik, and N.V. Barovskii, Phys. Rev. B 67, 140501 (2003).

3 J.R. Clem, Ann. of Phys. (NY) 40, 268 (1966).

${ }^{4}$ B.T. Geilikman Soviet Phys. JETP 21, 796 (1965); E.A. Pashitskii and V.M. Chernousenko, Soviet Phys. JETP 60, 1483 (1971); J. Ashkenazi and C.G. Kuper, Physica C 153-155, 1315 (1988).

5 H. Rietschel and L.J. Sham, Phys. Rev. B 28, 5100 (1983); S. Küchenhoff and P. Wölfle, Phys. Rev. B 38, 935 (1988).

6 J. Ruvalds, Adv. Phys. 30, 677 (1981).

7 Y. Takada, Phys. Rev. B 37, 150 (1988); 39, 11575 (1989); J. Phys. Soc. Jap. , 45, 786 (1978); 49, 1713 (1980); 61, 238 (1992).

8 Y. Takada, J. Phys. Soc. Jap. 45, 786 (1977)

9 W. McMillan and J. Rowell in Superconductivity, R. Parks Ed. (Dekker, New-York, 1969); E. Wolf Principles of Electron Tunneling Spectroscopy, (Oxford University Press, Oxford, 1985).

10 Y. Takada, J. Phys. Chem. Solids 54, 1779 (1993).

11 V.Z. Kresin, Phys. Rev. B 35, 8716 (1987).

12 V.Z. Kresin and H. Morawitz, Phys. Rev. B 37, 7854 (1988); J. Supercond. 1, 89 (1988); Phys. Lett. A 145, 368 (1990).

13 H. Morawitz, I. Bozovic, V.Z. Kresin, G. Rietveld, and D. van der Marel Z. Phys. B 90, 277 (1993).

14 A. Bill, H. Morawitz, and V.Z. Kresin, J. Low Temp. Phys. 117, 283 (1999); J. Supercond. 13, 907 (2000); Phys. Rev. B 66, 100501 (2002).

15 A.Abrikosov, L.Gor'kov, and I.Dzyaloshinskii, Methods of quantum field theory in statistical physics (Dover, New York, 1963); G.D. Mahan, Many-Particle Physics, (Plenum Press, New-York, 1993).

16 B. Geilikman, Sov. Phys. -USP. 18, 190 (1975).

17 P. Nozières and D. Pines, The Theory of Quantum Liquids, (Addison Wesley, 1989).

18 H. Tou, Y. Maniwa, T. Koiwasaki, and S. Yamanaka, Phys. Rev. Lett. 86, 5775 (2001); Phys. Rev. B 63, 020508 (2000).

19 G. Geilikman and V.Z. Kresin, Sov. Phys. Semiconductors 2, 639 (1968)

20 Y. Takada, Phys. Rev. A 28, 2417 (1983); 30, 3882 (1984).

21 A.L. Fetter, Ann. of Phys. (NY) 88, 1 (1974).

${ }^{22}$ C. Falter, G.A. Hoffmann, and F. Schnetgöke, J. Phys. C 14, 3239 (2002); C. Falter, M. Klenner, and G.A. Hoffmann, Phys. Stat. Sol. (b) 209, 235 (1998).

23 C. Falter and F. Schnetgöke, Phys. Rev. B 65, 054510 (2002).

24 F. Stern Phys. Rev. Lett. 18, 546 (1967).

25 C.S. Owen and D.J. Scalapino, Physica 55, 691 (1971).

26 S.Yamanaka, K-I.Hotehama, and H.Kawaji, Nature 392,
580 (1998); S. Yamanaka, H. Kawaji, K-I. Hotehama, and M. Ohashi, Adv. Mater. 8, 771 (1996).

27 H. Kawaji, K. Hotehama, and S. Yamanaka, Chem. Mater. 9, 2127 (1997).

28 S. Shamoto, T.Kato, Y.Ono, Y.Miyazaki, K.Ohoyama, M.Ohashi, Y.Yamaguchi, and T.Kajitani, Physica C 306, 7-14 (1998).

29 S. Shamoto, K. Iizawa, M. Yamada, K. Ohoyama, Y. Yamaguchi, and T. Katjitani, J. Phys. Chem. Solids 60, 1431 (1999).

30 P. Adelmann, B. Renker, H. Schober, M. Braden, and F. Fernandez-Diaz, J. Low Temp. Phys. 117, 449 (1999).

31 S. Yamanaka and H. Tou, Curr. Op. Sol. State Mat. Sci. 5, 545 (2001).

${ }^{32}$ H. Tou, Y. Maniwa, and S. Yamanaka, Phys. Rev. B 67, 100509 (2003).

33 T. Yokoya, Y.Ishiwata, S. Shin, S. Shamoto, K. Iizawa, T. Kajitani, I. Hase, and T. Takahashi, Phys. Rev. B 64, 153107 (2001).

34 A. Cros, A. Cantarero, D. Beltrán-Porter, J. Oró-Solé, and A. Fuertes, Phys. Rev. B 67, 104502 (2003).

35 R. Weht, A. Filippetti, and W.E. Pickett, Europhys. Lett. 48, 320 (1999).

36 I. Hase and Y. Nishihara, Phys. Rev. B 60, 1573 (1999).

37 W.A. Little, Phys. Rev. 134, A1416 (1964).

38 D. Jérome, A. Mazaud, M. Ribault, and K. Bechgaard, J. Physique Lett. 41, L95 (1980).

39 C.S. Jacobsen, D.B. Tanner, and K. Bechgaard, Phys. Rev. Lett. 46, 1142 (1981).

${ }^{40}$ H. Tajima, H. Kanbara, H. Kuroda, and G. Saito, Sol. State Comm. 57, 911 (1986).

41 H. Kino and H. Fukuyama, J. Phys. Soc. Jap. 64, 2726 (1995).

42 T. Ishiguro, K. Yamaji and G. Saito, Organic Superconductors, 2nd ed., edited by P. Fulde, Springer Series in Solid State Physics, vol. 88 (Springer, Berlin Heidelberg, 1998).

43 J. Singleton and C. Mielke, Contemp. Phys. 43, 63 (2002).

44 H. Urayama, H. Yamochi, G. Saito, S. Sato, A. Kawamoto, J. Tanaka, T. Mori, Y. Maruyama, H. Inokuchi, Chem. Lett. 3, 463 (1988).

45 C. Mielke, J. Singleton, M-S. Nam, N. Harrison, C.C. Agosta, B. Fravel, and L.K. Montgomery, J. Phys.: Condens. Matter 13, 8325 (2001).

46 Y-N. Xu, W.Y. Ching, Y.C. Jean, and Y. Lou, Phys. Rev. B 52, 12946 (1995).

47 A. Ugawa, G. Ojima, K. Yakushi, and H. Kuroda, Phys. Rev. B 38, 5122 (1988).

48 K.D.Carlson, A.M. Kini, R.A. Klemm, H.H. Wang, J.M. Williams, U. Geiser, S.K. Kumar, J.R. Ferraro, K.R. Lykke, P. Wurz, S. Fleshler, J.D. Dudek, N.L. Eastman, P.R. Mobley, J.M. Seaman, J.D.B. Sutin, and G.A. Yaconi, Inorg. Chem. 31, 3346 (2002); K.D. Carlson, J.M. Williams, U. Geiser, A.M. Kini, 
H.H. Wang, R.A. Klemm, S.K. Kumar, J.A. Schlueter, J.R. Ferraro, K.R. Lykke, P. Wurz, D.H. Parker, J.D.B. Sutin, J.E. Schirber, E.L. Venturini, and P. Stout, J. Am. Chem. Soc. 114, 10069 (1992).

49 A.M. Kini, K.D. Carlson, H.H. Wang, J.A. Schlueter, J.D. Dudek, S.A. Sirchio, U. Geiser, K.R. Lykke, and J.M. Williams, Physica C 264, 81 (1996); A.M. Kini, K.D. Carlson, J.D. Dudek, U. Geiser, H.H. Wang, and J.M. Williams, Synth. Met. 85, 1617 (1997).

50 L. Pintchovius, H. Rietschel, T. Sasaki, H. Mori, S. Tanaka, N. Toyota, M. Lang, and F. Steglich, Europhys. Lett. 37, 627 (1997).

51 S. Sugai, Phys. Rev. B 4714374 (1993).

52 A. Girlando, M. Masino, A. Brillante, R.G. Della Valle, and E. Venuti, Phys. Rev. B 66, 100507 (2002).

53 G. Varelogiannis, Phys. Rev. Lett. 88, 117005 (2002).

${ }^{54}$ H. Elsinger, J. Wosnitza, S. Wanka, J. Hagel, D. Schweitzer, and W. Strunz, Phys. Rev. Lett. 84, 6098 (2000).

55 I. Bozovic, private communication.

56 V.Z. Kresin and S.A. Wolf, Phys. Rev. 41, 4278 (1990).

57 V.Z. Kresin and S.A. Wolf, Fundamentals of Superconductivity, Plenum Press, New York (1992).

58 F.R. Gamble, F.J. Di Salvo, R.A. Klemm, and T.H. Geballe, Science 168, 568 (1970).

59 A. Bill, V. Hizhnyakov, D. Nevedrov, E. Seibold and E. Sigmund, Z. Phys. B 104, 753 (1997). 